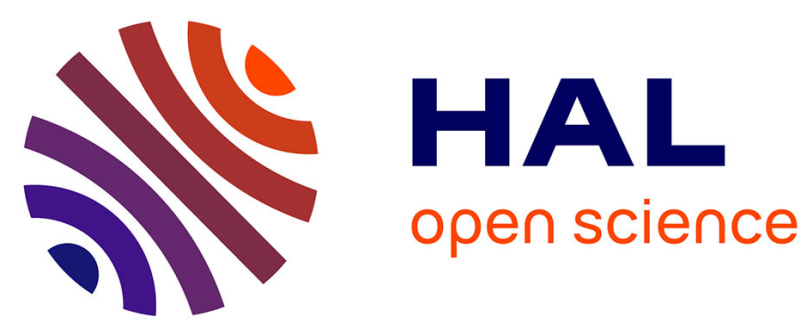

\title{
Towards a New Approach for Building Trust and Confidence in Machine Learning Data and Models: Based on an Integration of Artificial Intelligence and Blockchain (Part 1) \\ Hammou Fadili
}

\section{To cite this version:}

Hammou Fadili. Towards a New Approach for Building Trust and Confidence in Machine Learning Data and Models: Based on an Integration of Artificial Intelligence and Blockchain (Part 1). Journal of Applied Business and Economics, 2021, 22 (9), 10.33423/jabe.v22i9.3672 . hal-03093015

\section{HAL Id: hal-03093015 https://hal-cnam.archives-ouvertes.fr/hal-03093015}

Submitted on 1 Feb 2021

HAL is a multi-disciplinary open access archive for the deposit and dissemination of scientific research documents, whether they are published or not. The documents may come from teaching and research institutions in France or abroad, or from public or private research centers.
L'archive ouverte pluridisciplinaire HAL, est destinée au dépôt et à la diffusion de documents scientifiques de niveau recherche, publiés ou non, émanant des établissements d'enseignement et de recherche français ou étrangers, des laboratoires publics ou privés. 


\title{
Towards a New Approach for Building Trust and Confidence in Machine Learning Data and Models: Based on an Integration of Artificial Intelligence and Blockchain (Part 1)
}

\author{
Hammou Fadili \\ Laboratoire CEDRIC of CNAM \\ Fondation Maison Sciences de l'Homme
}

\begin{abstract}
Sentiments and opinions trends analysis and study are two important disciplines of Big Data exploitation. In our case related to Business Intelligence (BI), they make it possible to detect and extract relevant information from raw unstructured textual data, which we call: Textual Business Intelligence (TBI). It is in this context that we propose a new approach allowing to build trust and confidence in machine learning data and therefore in models: based on an integration and a convergence of artificial intelligence and Blockchain. This first part of the article will be dedicated to the motivation and the state of the art.
\end{abstract}

Keywords: artificial intelligence, blockchain, machine learning, neural models, sentiments analysis, text mining

\section{INTRODUCTION}

The current project is part of the natural evolution of the researches carried out in our main research domain in its general strategy on Business Intelligence (BI), and in its orientation relative to the exploitation of Big Data. In this context, data comes mainly from users/customers sentiments and opinions, expressed via social medias, forums, blogs, sales sites, etc., on all kinds of subjects such as events, products, attitudes, etc., to express their feelings and experiences.

Analysing sentiments and experiences of users is a major problematic for companies. Several studies and statistics confirm that more than $80 \%$ of Internet users consult the comments and opinions of former users before making their own purchases. Recommendations influence our opinions on products, services, etc. and therefore influence our purchases. Companies consequently have a great interest in understanding their customers feedback and comments. They must invest massively in intelligent systems allowing automatic sentiments analysis and opinions mining, in order to be helped to adapt to users' requirements and improve their profits; in the new open world of business and economic intelligence which is the web. Despite advances in this area, several recent studies show that only $29 \%$ of companies use data in their decision. This is due to several problems, mainly related to the nature of the existing solutions which are not yet mature enough, and to their costs, which are still too expensive to implement.

The phenomena of business data analysis in the context of "Big Data", for intelligence purposes, concerned mainly the static aspects of textual data models.

However, due to their nature in this context, data as well as their characteristics are "naturally" dynamic and therefore their handling is necessary. The analysis of dynamic aspects of data such as the study \& 
extraction of indicators and the analysis \& prediction of their behaviour over time, has become a major issue encouraging the emergence of new research projects, moreover difficult and complex. This complexity can be amplified if data additionally includes specific information such as anomalies, fake information, etc. In fact, the latter, which can alter the judgment and the perception that we can have on the real facts, must be considered in the treatment processes. Detecting and managing these elements is an important task for the dynamic data analysis and for the impact on the associated target indicators trends.

It is in this context that we carried out the current research project, on the dynamic data analysis, entitled:

"Designing a new approach to build trust and confidence in machine learning data and models: based on an integration and the convergence of artificial intelligence and Blockchain"

In this project, we are interested in the issues of detecting anomalies \& false information and their causes in the dynamic sentiments influencing the trends prediction in associated time series/sequences, by proposing a solution, which can limit them, based on a security, certification, etc. data system, via Blockchain.

The main objectives of the project can be recalled as follows:

- Minimize the role and the intervention of a human user, in the context of big data; where processing by a human being is almost impossible to perform (large amount of data). The system should be able to "extrapolate" by exploiting a minimum of annotated $\&$ classified (pretreated) data and learn by it itself the latent science contained in the text, in order to extract all the useful information for decision-making.

- Design and develop approaches considering the dynamic aspects of data.

- Design and develop approaches to detect anomalies and fake information in data.

- Model the prediction of targeted indicators behaviour considering anomalies and fake information.

- Study a solution that can prevent fake information, build confidence and trust in data and make reliable analysis and predictions.

\section{STATE OF THE ART}

In the following, we will present a state of the art of existing knowledge on dynamic data behaviour analysis for sentiments analysis in the context of Big Data. We will describe the state of work there, according to the following:

- Approaches of dynamic sentiments and opinions analysis and prediction,

- Anomalies and fake information detection in considered data,

- Domains of use of Blockchain technology.

These elements will allow us, thereafter, to note that these three types of technologies evolve independently, on the one hand, on the other hand, to highlight the gaps in the current state of existing knowledge, on:

- Integration of fake information in the data analysis process,

- Building certified and trusted data,

- Predictions reliability.

\section{Dynamic Sentiments Analysis and Prediction}

Dynamic sentiments analysis consists in observing in time, polarities trends relating to subjective elements (words and sentences which express opinions, sentiments, emotions ...) to decide on the orientation of a document (Turney, 2002). 
Several works have been devoted to the analysis and prediction of dynamic sentiments. They consider a sentiment as a tendency to feel an emotion in relation to an object or in relation to a person. It also concerns people's opinions, their assessments and their feelings towards entities, events, etc. Most of the works dealing with dynamic aspects of sentiments in the context of Big Data, deal with sentiments expressed in writing through comments on products, websites, blogs, discussion forums, etc. The exchanges being naturally dynamic, constitute a favourable context influencing and modifying the messages and the sentiments conveyed. Therefore, a sentiment, an opinion, etc. cannot be static, they are dynamic and evolve over time, depending on the evolution of their context (opinions of others, exchanges, etc.).

In terms of approaches and implementations, several studies have been conducted in this area; we can cite for example. The analysis of these works allowed us to deduce two classes of approaches depending on the nature of the elements on which the prediction relates:

- Approaches based on the prediction of temporal sequences: in this case the prediction is made directly from the contents or from their final polarities of past times. The polarities can be calculated at the level of words, sentences, paragraphs, texts, etc.

- Approaches based on the prediction of time series: the prediction is made from the numerical values calculated and extracted from the analysed contents.

In both cases, and if we refer to the complete definition of the sentiments analysis (Liu and Zang 2012), relating to the automatic identification of 4 components of a sentiment, namely, the entity to which relates the sentiment, the aspect (property) on which the judgment relates, the owner or the emitter of the sentiment and finally the sentiment itself, the studied approaches are incomplete. Indeed, they are only interested in the detection and/or prediction of the sentiments and their polarities alone, neglecting other characteristics, namely: the automatic detection of the entity to which the sentiment relates, the identification of the author and the characterization of the evaluated aspect.

In addition, in terms of considering the data quality on which the analysis and prediction relate, we have found that:

- Not all the studied approaches deal with the nature and the quality of data. No questions about the origin of data, or its integrity (if data has not been modified, falsified, etc.).

- In the case where we have fake information, no solutions can take it into account in their prediction process. Fake information is not considered to correct predictions.

\section{Detection and Integration of Fake Information}

Much of the sentiments analysis is based on text mining. It can target the study and detection of sentiments, opinions, emotions and subjectivities in text (Pang et al. 2004); in relation to a specific area such as: advertising, marketing, production, business, politics, psychology, etc.

It is obvious to note that these fields are very sensitive to the tendencies of comments and exchanges between users which can considerably influence the sentiments and opinions conveyed in the said contents.

Detecting fake information has become a major area of research. Several works have been devoted to the treatment of fake information, mainly in social medias. In these studies, it is shown that considering fake information can cause changes in messages behaviours. It is a simple way that people and organizations propaganda use to redirect trends in messages, events, etc. as evidenced by various campaigns damaging reputation, elections, ...

In terms of processing, most approaches exploit statistical and classification methods to study, analyse and detect fake information; but none of them makes it possible either to prevent them or to study their impact on the evolution of dynamic aspects of the processed data, in this case on the sentiments trends.

\section{Domains of Use of Blockchain}

The studies carried out in for example, show that Blockchain is a new emerging technology of network storage and transfer protocols, transparent and secure, based on peer-to-peer networks without a central control. The computer model of Blockchain consists of sequences of ordered transactions grouped by blocks. Transactions are created and issued by one or more network identities: 
- Secured

- Encrypted,

- Consistent according to specific constraints (Smart Contracts)

- Controlled by consensus of network elements

- Recorded on some sort of shared registry

- Hardly modifiable and alterable.

FIGURE 1

BLOCKCHAIN STRUCTURE

\begin{tabular}{|c|c|c|c|}
\hline block $\mathrm{n}$ & block $n+1$ & block $n+2$ & block $n+3$ \\
\hline Header $n$ & Header $n+1$ & Header $n+2$ & Header $n+3$ \\
\hline Hash n-1 & Hash n & Hash n+1 & lash \\
\hline Transaction a & Transaction b & Transaction c & Transaction d \\
\hline Transaction a+1 & Transaction $b+1$ & Transaction c+1 & Transaction $\mathrm{d}+1$ \\
\hline Transaction $a+2$ & Transaction $b+2$ & Transaction c+2 & Transaction $d+2$ \\
\hline & $\begin{array}{l}\cdots \\
-\cdots\end{array}$ & $\mid$\begin{tabular}{l}
$\cdots$ \\
\hdashline$-\ldots+\ldots-$
\end{tabular} & - \\
\hline $\begin{array}{l}\text { Proof } x \text { of } n \\
\text { Proof } y \text { of } n \\
\text { Proof } z \text { of } n \\
\ldots\end{array}$ & $\begin{array}{l}\text { Proof } x \text { of } n+1 \\
\text { Proof } y \text { of } n+1 \\
\text { Proof } z \text { of } n+1 \\
\ldots\end{array}$ & $\begin{array}{l}\text { Proof } x \text { of } n+2 \\
\text { Proof } y \text { of } n+2 \\
\text { Proof } z \text { of } n+2 \\
\ldots\end{array}$ & $\begin{array}{l}\text { Proof } x \text { of } n+3 \\
\text { Proof } y \text { of } n+3 \\
\text { Proof } z \text { of } n+3 \\
\text {. }\end{array}$ \\
\hline Hash n & Hash & Hash $n+2$ & Hash $n+3$ \\
\hline
\end{tabular}

Current studies and implementations of Blockchain technology mainly concern implementations of cryptocurrencies, including Bitcoin and Ethereum. We even tend to believe that this technology can only be used for virtual currencies.

Other solutions go as far as implementing the concept of "Smart Contracts" which allow executing transactions implementing contracts between the different actors of Blockchain, via remuneration systems with tokens and/or virtual currencies.

These first implementations of this new technology were made in limited areas; while its major interest lies in its potential applications in several other domains and for many other needs; is that we will show in the current article.

\section{Sentiments Analysis and Prediction in a Multilingual Context}

The study of the several existing solutions in the domain of sentiments analysis and prediction, allowed us to conclude that:

- The sentiments analysis in a multilingual context is an unavoidable problem in that domain.

- The popularity of Internet use worldwide, generates a lot of content in social medias, blogs, forums, etc. in most world written languages, having no resources \& tools, no processed data, no corpus... allowing their automatic processing; makes this task difficult.

- Most of studied approaches only consider one language at a time, in this case English, in their analysis process. This is evidenced by the large number of resources and all tools available for English; but not numerous enough or non-existent for other languages.

\section{Conclusion}

Sentiment analysis is an emerging and very important discipline of "textual BI" and the use of big data. Although there are several proposals and approaches in the literature, there are few that deal with dynamic aspects and changes in data. In addition, the latter do not consider all the requirements of dynamic sentiments trends.

More concretely, from the present state of the art, we can deduce that the studied approaches: 
- Do not exhaustively model the concepts of context notion of dynamic data (dynamic context).

- Do not implement these concepts in processing, especially in machine learning systems.

- Do not allow to detect anomalies and fake information in data.

- Do not prevent fake information.

- Do not consider fake information to predict the behaviour of targeted indicators.

- Do not consider the notion of dynamic data quality: certification, trust, security, etc.

\section{MOTIVATION}

The analysis and study of dynamic sentiments are two important disciplines of the exploitation of big data. They make it possible to obtain information from raw textual data: Business Intelligence (BI) from textual data, we call: Textual Business Intelligence. Although there are several proposals and approaches in the literature, but there is no solution that can consider all aspects of sentiments analysis and especially their dynamic aspects: aspects difficult to model, to capture and to treat. In addition, if we consider the data quality (certification, trust, security, etc.), these analysis and prediction tasks become even more complex, incomplete and difficult to implement.

These numerous limitations are due to several problems, which we will detail later in this section; the main ones of which express and constitute the motivation of this work.

\section{Scientific \& Technological Problems Related to the Characterization of Dynamic Aspects of Sentiments}

Although the dynamic aspect is obviously very important in sentiments analysis, its management remains very limited; as evidenced by most studies and researches of the domain. Elements that can influence the behaviour of a sentiment are difficult to capture, especially by automatic processes. For example, following interactions, sentiments and opinions that users may have on a subject can change over time. The question is how to model and capture this influence? That constitutes one problem of the present work.

All the elements that can influence our sentiments, opinions... on subjects, constitute an important new notion, we call "dynamic context". In automatic processes, detecting the evolution of a sentiment, conditioned by the context changes, is a very complex task; requiring a complete modelling of the dynamic context and the dynamic aspects of sentiments, on the one hand, and on the other hand, the temporal modelling of natural language processing phenomena and of machine learning systems.

Defining all these aspects to enrich the dynamic context and to increase the efficiency of the associated processing systems is another challenge to be met.

\section{Challenges Related to the Prediction's Quality Based on Fake Information Management}

Obtaining a reliable analysis and therefore a reliable prediction from data supposes that data is reliable and not falsified. Companies and institutions regularly suffer from disinformation campaigns aimed at damaging their image and reputation. Consequently, one aspect of analyses and predictions quality depends on the presence or absence of fake information in the processed data. Their detection and prevention being very importance, will be integrated and considered in our approach.

From an automatic processing point of view, the detection of fake information is based on anomaly detection technologies which is a known discipline. Their detection depends on their representation and their characterization properties (see below); which requires for each kind, important adaptations in machine learning systems functions: regressions and/or classifications. The distinction between different aspects of fake information (for example: satire, parody, propaganda, etc.) [29] and the treatments adaptation for each aspect, constitute another major challenge.

The characteristics of fake information are normally continuous notions in their representation spaces. Switching to discrete polarized spaces representations is a task obviously resulting in a lot of information loss; during projections on the anomalies axes (true, false, anomaly rate, type, etc.). The loss of such 
information is another challenge in the field of sentiments prediction and its minimization (loss) constitutes a major technological problem to be solved.

Integrating fake information into the treatments process is essential for sentiments analysis and prediction. Obviously, this increases accuracy and improves performance. This is equivalent to inserting a pre-processing step allowing the detection and extraction of fake information from time units (which are blocks in our case, where the implementation is based on the Blockchain). This raises several questions reflecting the following technological problems to be solved:

- How to consider fake information in processing?

- How to pre-treat fake information?

- How to consider the results of pre-processing in global process?

\section{Scientific and Technological Problems Related to the Prevention of Fake Information Guaranteeing} Data Quality: Certification, Trust, Security, Etc.

This part is devoted to the scientific obstacles to be overcome to guarantee the quality of data on which the prediction relates. This, concerns mainly, the prevention of fake information, on the one hand, and on the other hand, security, certification and trusting data. These are unexplored disciplines which constitute major scientific problems for the present work.

Technologically, since the solution we are proposing is based on Blockchain technology (because it appears today to be the most suitable); its implementation in the context of sentiment analysis and prediction has never been studied. This constitutes another technological issue and its impact constitutes another problem for the present work.

\section{Scientific and Technological Problems Related to the Integration of AI and Blockchain}

Blockchain like artificial intelligence are recent and interesting technologies, even essential in some cases. The different implementations of Blockchain and artificial intelligence show that there is no integration of these technologies together. Studying solutions to demonstrate the complementarity of these technologies constitutes a major scientific issue.

This mutual complementarity implies two potential technological implementations following two axes:

- AI implementation at Blockchain nodes for smart and optimized management via distributed calculations.

- Blockchain exploitation to generate authentic, secure, certified, etc. data and models, thus avoiding data manipulation, censorship, etc.; able to control and redirect opinions by introducing and spreading fake information.

Implementing this dual integration constitutes a major technological problem to be overcome.

\section{Problems Related to Prediction Processing in a Multilingual Context}

The popularity of Internet use worldwide, generates a lot of content in social medias, blogs, forums, etc. in most of written languages of the world, some of which do not have resources, processed data, or corpora... allowing their automatic processing. Therefore, the semantic analysis in a multilingual context is an unavoidable problem for sentiments prediction.

Most of studied approaches consider only one language at a time, in this case English, in their analysis process. This is evidenced by the large number of resources and all available tools for English; but not numerous enough or nonexistent for other languages.

To address this problem, some approaches try to translate source documents into English to take advantage of available resources for that language. For us, this constitutes a limitation, because there is a risk of information loss during translation, which can have a negative impact on the prediction as for the polarity calculations.

Dealing with the multilingualism dimension in analysis and prediction process is a major problem that we must face. 


\section{Problems Related to Prediction Management in the Context of Big Data}

As with sentiments analysis, for predicting temporal trends of sentiments, pretreated data is essential in automatic data processing systems. They are used to model application domains such as ontologies, which express rules systems for reasoning and annotated training datasets for machine learning systems. However, the massive data generated in Big Data is raw, unstructured and unprocessed, therefore unusable by conventional automatic processes. This is a major obstacle for the analysis and therefore for the prediction, this is another important problem.

The integration of Big Data requirements into our solution is essential: it is an important element in our approach. In this context, user sentiments behavior prediction comes up against several difficulties:

- Lack of description rules for all possible uses

- Lack of preprocessed data in several domains

- Large amounts of data

Heterogeneous nature of the data

Unstructured nature of the data

\section{Deficiencies Related to the Exploitation of Advanced Artificial Intelligence Technologies: Memories and Latent Science Learning}

From our previous studies, we can easily deduce that most of existing technologies exploit the words of the text without considering either their meanings or their orders (bag of words). This is evidenced by the most used technology in this field, "Word Embeddings" or "Word2Vec" and its various implementations (SKIP-GRAM \& CBOW), which does not "code" either the meaning of words or their order in the representation vectors. It is obvious that the loss of the order implies "automatically" the loss of semantics: we know several sentences, where the meaning changes when we change the order of words in the sentence. Designing technological solutions considering the meaning and the order of words and especially the time order of blocks for prediction, bringing considerable improvements, is a first major technological problem for this section to be overcome.

Another problem encountered from the exploitation of deep learning architectures in sentiments analysis domain and therefore in prediction is that of fixed size of networks. A classic neural network configured and trained keeps its parameters throughout its life cycle. This poses problems in the case of sentences, paragraphs, texts, etc. composed of variable numbers of words. That's why, we exploited NGRAMS and sliding windows of fixed sizes to circumvent this problem, despite the loss of information due to the fact that the meaning of a word is linked to the phrase and sentence containing it and not to a window of words surrounding it. Developing technological solutions that can remedy this problem is another technological problem to be overcome. Note that this problem also had a decisive impact in the choice of our solution by adopting approaches based on an improved variant of recurrent neural networks, independent of the number of words to be processed and the length of sentences and documents.

Also, by deep learning, we mean multi-layer architectures, which in some cases can be composed of many layers. We can demonstrate mathematically that given that:

- The training of the network is done by optimizing the parameters minimizing the prediction error, by BACKPROPAGATION OF THE ERROR.

- The backpropagation is done by GRADIENT DESCENT by modifying the coefficients (system parameters) as a function of the error rate in the opposite direction of the greatest slope of the gradient error.

- In the case of a recurrent network, learning is calculated by MULTIPLYING THE COEFICIENTS of a layer by the SAME NUMBER $\lambda$ as many times as the rank of the layer in the network.

So,

- If the NUMBER $\lambda$ is less than 1, the learnings of the first layers are multiplied by a number close to zero which stop learning. In this case we are talking about the Vanishment of Gradient 
- If the NUMBER $\lambda$ is greater than 1, the learnings of the first layers are multiplied by a high number which stop learning. In this case, we are talking about the Explosion of Gradient

We have implemented an improved Long Short-Term Memory (LSTM) architecture to remedy these problems. But in the case of predicting time series, this architecture can also have limitations. We use the output of the next block for learning, correcting and finishing the prediction calculated by the current block in the past, i.e. in the period of the past time chosen for learning [t-p1, t]. Thanks to this optimized unsupervised learning, we can improve the prediction of the chosen future period. This is what we will try to show and test in our approach.

Addressing this is another important technological problem.

\section{Conclusion}

Given the magnitude of the problems described in this part relating to the modeling of dynamic sentiments and their contexts, fake information and their integration, technological choices and their implementation; only one part has been treated, the other part constitutes the perspectives of future work.

\section{CONCLUSION \& PERSPECTIVES}

Sentiment analysis has become a very important discipline in the exploitation of big data. Although there are several proposals and approaches in the literature, there is today no solution which can consider all the requirements of life cycles of sentiments prediction as well as the associated treatments.

It is in this context that we proposed a solution improving the dynamic aspects of the following elements:

- The disambiguation at the beginning of the process

- The context to deduce the global semantics and thereafter the subjectivity and the polarity

- The possibility of ontology integration and other knowledge bases to solve problems such as: acronyms, text messages, expressions (expressions), etc.

- The implementation of an improved LSTM

- Support of intermediate predictions to improve overall prediction

- Learning and correcting predictions thanks to Blockchain

- Guaranteeing the data quality and predictions thanks to Blockchain

Following various tests, we can conclude: the more properties available characterizing the global context for the instances, the more reliable the system is in its predictions. The exhaustive definition and the extraction of the Characteristics being complex cannot be done without difficulties, several limitations were encountered, and several were abandoned as explained previously. As a reminder, we have:

- Used and abandoned solutions based on descriptions such as ontologies; because they are difficult to set up. In fact, we have been talking about ontologies for several years, but today there are no complete ontologies, except in very limited fields. Therefore, we redirected our work towards unsupervised or mixed solutions for instantiating our extended models.

- Used and abandoned technologies based on "sparse" vector representations; because they cause combinatorial explosions in treatments. We have adopted the "word embeddings" model: new "revolutionary" representations that can be learned from corpus and projected into "semantic" reduced dimensions vector spaces (around 300). This technology was also abandoned in its initial version, because the representations are ambiguous. Therefore, we have improved the latter by training them on the meaning and not directly on words.

- Tested and abandoned several types of algorithms and classifiers, because they did not meet our expectations. Therefore, we adopted and adapted the model of recurrent neural networks (LSTM).

From the current state of progress of the project, it can be said that only part of the problems described above has been treated and solved, the other part constitutes the perspectives for this work. We can recall some of them (those which seem strategic and/or important to us): 
- Blockchain technology is very recent, we tried a very first exploitation, deepening its study in order to integrate it more in our processes is a major strategic axis of the perspectives of this work.

- Also, the integration of Blockchain and artificial intelligence seems promising, we have also tried a very first integration of these two technologies of the future. Deepening this study is also another major strategic axis of the perspectives of this work.

- Deepen the study of the characteristics: irony, humour (depending on the culture), metaphor, expressions, etc.; as well as their integration into the process of sentiments analysis and prediction.

- Consider multi-domain and multi-language aspects in sentiments analysis process.

- Implement mechanisms preventing the re-identification of people according to their opinion, comments, etc. many scandals where people have been re-identified from their comments. This question poses an even wider problem on the re-identification of people in Big Data. This is another more important issue of our research.

- Consider nuances of subjectivity which can be expressed through different concepts (Affect, feeling, emotion, and opinion). The distinction between these different aspects and the adaptation of treatments for each constitutes another axis of the perspectives of this work.

- Another perspective concerns the extension of sentiments analysis to other types of data, such as audio and video (multimodal sentiment analysis).

- The integration of the approach in the context of Big Data and its constraints. We have optimized part of the treatments and have dealt with the "unstructured data" and "unavailability/lack" aspects of the processed data, characterizing Big Data, but without considering all its other constraints: acquisition, storage, parallel processing, etc. Taking on all the constraints of Big Data is another perspective of our work.

- Finally, development of a unified system implementing the global "workflow". As explained above, the testing process was done using separate modules and platforms. We therefore envisage the development of other IT modules dedicated to specific tasks, which can be integrated into a single standalone application implementing the entire "Workflow" data processing.

\section{REFERENCES}

Allcott, H., \& Gentzkow, M. (2017). Social media and fake news in the 2016 election. Journal of Economic Perspectives, 31(2), 211-36.

Azaria, A., Ekblaw, A., Vieira, T., \& Lippman, A. (2016, August). Medrec: Using blockchain for medical data access and permission management. In Open and Big Data (OBD), International Conference (pp. 25-30). IEEE.

Balmas, M. (2014). When fake news becomes real: Combined exposure to multiple news sources and political attitudes of inefficacy, alienation, and cynicism. Communication Research, 41(3), 430454.

Cachin, C. (2016, July). Architecture of the hyperledger blockchain fabric. In Workshop on Distributed Cryptocurrencies and Consensus Ledgers (Vol. 310).

Del Vicario, M., Quattrociocchi, W., Scala, A., \& Zollo, F. (2018). Polarization and fake news: Early warning of potential misinformation targets. arXiv preprint arXiv:1802.01400.

Esuli, A., \& Sebastiani, F. (2007). SentiWordNet: a high-coverage lexical resource for opinion mining. Evaluation, 17, 1-26.

Frijda, N.H., Mesquita, B., Sonnemans, J., \& Van Goozen, S. (1991). The duration of affective phenomena or emotions, sentiments and passions. 
Ghiassi, M., Skinner, J., \& Zimbra, D. (2013). Twitter brand sentiment analysis: A hybrid system using ngram analysis and dynamic artificial neural network. Expert Systems With Applications, 40(16), 6266-6282.

Juang, C.F., Ku, S.J., \& Huang, H.J. (2004, October). Fuzzy temporal sequence processing by recurrent neural fuzzy network. In Systems, Man and Cybernetics, 2004 IEEE International Conference (Vol. 6, pp. 5847-5851). IEEE.

Lazer, D.M., Baum, M.A., Benkler, Y., Berinsky, A.J., Greenhill, K.M., Menczer, F., . . Schudson, M. (2018). The science of fake news. Science, 359(6380), 1094-1096.

Marchi, R. (2012). With Facebook, blogs, and fake news, teens reject journalistic "objectivity". Journal of Communication Inquiry, 36(3), 246-262.

Miller, G.A. (1995). WordNet: a lexical database for English. Communications of the ACM, 38(11), 3941.

Mozer, M.C. (1993, February). Neural net architectures for temporal sequence processing. In Santa Fe Institute Studies in the Sciences of Complexity-Proceedings Volume (Vol. 15, pp. 243-243). Addison-Wesley Publishing Co.

Pérez-Rosas, V., Kleinberg, B., Lefevre, A., \& Mihalcea, R. (2017). Automatic detection of fake news. arXiv preprint arXiv: 1708.07104.

Pilkington, M. (2016). 11 Blockchain technology: principles and applications. Research Handbook on Digital Transformations, 225.

Rubin, V.L., Conroy, N.J., \& Chen, Y. (2015, January). Towards news verification: Deception detection methods for news discourse. In Hawaii International Conference on System Sciences.

Shand, A.F. (1920). The foundations of character: Being a study of the tendencies of the emotions and sentiments. Macmillan and Company, limited.

Shao, C., Ciampaglia, G.L., Varol, O., Flammini, A., \& Menczer, F. (2017). The spread of fake news by social bots. arXiv preprint arXiv:1707.07592, 96-104.

Shu, K., Sliva, A., Wang, S., Tang, J., \& Liu, H. (2017). Fake news detection on social media: A data mining perspective. ACM SIGKDD Explorations Newsletter, 19(1), 22-36.

Sruk, V., \& Gole, P.A. (2017). ICSD 2017. BELGRADE FACULTY OF MANAGEMENT, METROPOLITAN UNIVERSITY. Retrieved from www.icsd.metropolitan.ac.rs, 40.

Swan, M. (2015). Blockchain: Blueprint for a new economy. O'Reilly Media, Inc.

Tandoc, E.C., Jr., Lim, Z.W., \& Ling, R. (2018). Defining "fake news" A typology of scholarly definitions. Digital Journalism, 6(2), 137-153.

Teo, K.K., Wang, L., \& Lin, Z. (2000, November 18-21). Wavelet multi-layer perceptron neural network for time-series prediction. In Proc. 10th International Conference on Computing and Information. ICCI 2000, Kuwait.

Wörgötter, F., \& Porr, B. (2005). Temporal sequence learning, prediction, and control: a review of different models and their relation to biological mechanisms. Neural Computation, 17(2), 245319.

Yang, Y., Zheng, L., Zhang, J., Cui, Q., Li, Z., \& Yu, P.S. (2018). TI-CNN: Convolutional neural networks for fake news detection. arXiv preprint arXiv:1806.00749.

Zhou, X., Tao, X., Yong, J., \& Yang, Z. (2013, June). Sentiment analysis on tweets for social events. In Computer Supported Cooperative Work in Design (CSCWD), 2013 IEEE 17th International Conference (pp. 557-562). IEEE.

Zhu, Q., Chen, J., Zhu, L., Duan, X., \& Liu, Y. (2018). Wind Speed Prediction with Spatio-Temporal Correlation: A Deep Learning Approach. Energies, 11(4), 705.

Zyskind, G., \& Nathan, O. (2015, May). Decentralizing privacy: Using blockchain to protect personal data. In Security and Privacy Workshops (SPW), 2015 IEEE (pp. 180-184). IEEE. 